\title{
Quantum states and specific heat of low-density He gas adsorbed within the carbon nanotube interstitial channels: Band structure effects and potential dependence
}

\author{
Antonio Šiber国 and Hrvoje Buljan² \\ 1 Institute of Physics, P.O. Box 304, 10001 Zagreb, Croatia \\ ${ }^{2}$ Department of Physics, Faculty of Science, University of Zagreb, PP 332, 10001 Zagreb, Croatia
}

\begin{abstract}
We calculate the energy-band structure of a He atom trapped within the interstitial channel between close-packed nanotubes within a bundle and its influence on the specific heat of the adsorbed gas. A robust prediction of our calculations is that the contribution of the low-density adsorbed gas to the specific heat of the nanotube material shows pronounced nonmonotonic variations with temperature. These variations are shown to be closely related to the band gaps in the adsorbate density of states.
\end{abstract}

PACS numbers: 65.80.+n, 68.43.De, 68.65.-k

*Corresponding author. E-mail: asiber@ifs.hr 


\section{INTRODUCTION}

The adsorption of gases in and on the bundles of carbon nanotubes has recently attracted

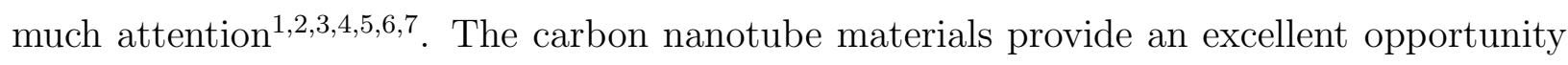
for a study of adsorption $\mathrm{l}$ in confined geometries. This phenomenon is not only of academic interest - a motivation for its study is potential application of the carbon nanotube materials for a very efficient storage of gases]国到.

${ }^{4} \mathrm{He}$ atoms adsorbed in strongly bound positions have been detected by measurements of desorption from single wall nanotube (SWNT) bundlest. Recent low temperature specific heat measurements on SWNT bundles 10 have detected a contribution to the specific heat which is very sensitive to the amount of adsorbed gases, in particular ${ }^{4} \mathrm{He}$. Motivated by these experimental results, here we theoretically analyse the behavior of He atoms adsorbed in the samples consisting of SWNT bundles. In particular, we investigate the quantum states available for the adsorption of He atoms in the interstitial channels between the SWNTs, close-packed in a triangular lattice. We analyse the consequences of specificities of energy spectrum of adsorbed He atoms on the specific heat pertaining to the adsorbed He gas.

The effects of interactions between interstitial He adsorbates and their consequences on the specific heat have been examined in Ref. 3. Transition of He gas adsorbed in the interstitial channels to an anisotropic condensed phase has been predicted as a result of attraction between atoms in neighboring channels. In this work we concentrate on the interaction of He atoms with a surrounding nanotube medium and neglect He-He interactions.

The main results and the outline of the article are as follows. In Sec. 2, we propose a model potential for the interaction of He atoms with surrounding medium. Our model of the potential confining He adsorbate to an interstitial channel is sufficiently refined to allow the investigation of the influence of details of He confinement on the specific heat of adsorbate gas. We shall demonstrate that the periodicity of the potential along the channel axis causes gaps in the adsorbate density of states. These gaps increase with the magnitude of corrugation of the interaction potential along the interstitial channel axis. As 
a consequence, in Sec. 3 we shall show that the specific heat pertaining to the adsorbate gas shows nonmonotonic behavior with temperature. The precise dependence of specific heat on the temperature will be shown to be determined by the corrugation of the interaction potential.

Our approach is similar in spirit to the one presented in Ref. 15, where the specific heat of low-density He films on graphite has been examined. The most important results of this article are summarized in Sec. 4.

\section{ADSORPTION POTENTIAL AND BAND STRUCTURE CALCULATION}

The Schrödinger equation for the wavefunction $\Psi_{\kappa}(x, y, z)$ of a single adsorbate within the interstitial channel is

$$
\left[-\frac{\hbar^{2}}{2 M} \nabla^{2}+V(x, y, z)\right] \Psi_{\kappa}(x, y, z)=E_{\kappa} \Psi_{\kappa}(x, y, z),
$$

where $M$ is the mass of adsorbate and $\kappa$ denotes a set of quantum numbers needed for the specification of the quantum state of the adsorbate.

To find the quantum states supported by equation (1) one needs to construct the potential $V(x, y, z)$. However, the construction of the relevant potential is somewhat problematic for two reasons. First, the potential describing the interaction of He atom with the carbon nanotube in not known with great precision and often the model potentials based on the assumption of pairwise additivity of binary He-C interactions are used国. Second, the details of arrangement of nanotubes within a bundle are also not known. In particular, the relative axial alignment of nanotubes (relative axial offsets) within a bundle is not known la. As shown in Ref. 2, different nanotube alignments can produce very different potentials for adsorption of He atom in the interstitial channels. It is also likely that bundles contain a mixture of tubes with different wrapping angles11,12. Details of this mixture may vary depending on a particular bundle in the sample. Furthermore, the distribution of nanotube diameters in the sample has a finite width 13,14 . All these facts imply that a detailed information on the potential is at present not available and that the relevant potential depends on the interstitial channel in question. 
Here we propose a model potential $V(x, y, z)$ which captures all the essential physics involved, yet simple enough to allow an efficient and transparent calculation of the quantum states and predict the dependence of specific heat on temperature. According to references t and 5, the effective potential confining the motion of He atom in the plane perpendicular to the channel axis can be represented by a paraboloid-like shape with the minimum at the geometrical center of the channel (see Fig. 1 of Ref. 4 ). The potential varies along the channel axis, reflecting the variations of the nanotube electronic density in this direction $\mathrm{G}$. The simplest potential which displays the described behavior is

$$
\begin{aligned}
V(x, y, z) & =D+\alpha\left(x^{2}+y^{2}\right)+2 V_{g}(x, y) \cos (g z) \\
& =V_{0}(x, y)+2 V_{g}(x, y) \cos (g z)
\end{aligned}
$$

where $g=2 \pi / a$ is the inverse lattice vector associated with the periodicity of the potential along the channel axis ( $z$ direction) and the factor of 2 in front of $V_{g}(x, y)$ term has been introduced for convenience. The origin of $x$ and $y$ axes is positioned at the center of the channel and the channel axis is perpendicular to the $x y$ plane. $D$ defines the minimum of the potential at the center of the channel, in absence of the corrugation term, $V_{g}(x, y)$. Equation (11) can be solved by writing the wavefunction as

$$
\Psi_{\lambda}^{k}(x, y, z)=\sum_{m, n, G} \beta_{m, n, k+G}^{\lambda} \Phi_{m, n, k+G}(x, y, z)
$$

where

$$
\begin{aligned}
\Phi_{m, n, k+G}(x, y, z) & =\frac{\delta}{\sqrt{\pi 2^{m+n} m ! n ! L_{z}}} H_{m}(\delta x) H_{n}(\delta y) \exp \left(-\frac{1}{2} \delta^{2}\left(x^{2}+y^{2}\right)\right) e^{i(k+G) z} \\
& =\mid m, n ; k+G>
\end{aligned}
$$

is the solution of the hamiltonian containing only $V_{0}(x, y)$ part of the potential. Here, $L_{z}$ is the box quantization length along the axis of the channel, $H_{m, n}$ are the Hermite polinomials, $\delta^{4}=2 M \alpha / \hbar^{2}, m$ and $n$ are the harmonic oscillator quantum numbers, $k$ is the wavevector associated with the motion of He atoms in $z$ direction, and $G$ is a reciprocal lattice vector given as $G=p g$, with $p$ integer, associated with the periodicity of the potential in $z$ direction. The Schrödinger equation (1) reduces to matrix equation 


$$
\begin{aligned}
& \sum_{m^{\prime}, n^{\prime}, G^{\prime}}\left[E_{m^{\prime}, n^{\prime}}+\frac{\hbar^{2}}{2 M}\left(k+G^{\prime}\right)^{2}-E_{\lambda}\right] \delta_{m, m^{\prime}} \delta_{n, n^{\prime}} \delta_{G, G^{\prime}} \beta_{m, n, k+G}^{\lambda} \\
+ & \sum_{m^{\prime}, n^{\prime}, G^{\prime}}<m, n ; k+G\left|2 V_{g}(x, y) \cos (g z)\right| m^{\prime}, n^{\prime} ; k+G^{\prime}>\beta_{m, n, k+G}^{\lambda}=0 .
\end{aligned}
$$

$E_{m^{\prime}, n^{\prime}}$ are the eigen energies of the hamiltonian without the corrugation part of the potential and are given as

$$
E_{m^{\prime}, n^{\prime}}=D+\hbar \omega_{0}\left(m^{\prime}+n^{\prime}+1\right)
$$

where $\omega_{0}=\sqrt{2 \alpha / M}$ is the characteristic frequency of the harmonic oscillator. The spectrum of energies of adsorbed He atom can be found by looking for nontrivial solutions of equation (5). For fixed $k$, this produces a number of different solutions (bands) denoted by index $\lambda$.

By systematic examination of a range of $k$ values, it is possible to obtain the full band structure, $E_{\lambda}(k)$. Similar approach has been used for a calculation of band structure of He atoms adsorbed on the surface of graphite 15 and $\mathrm{NaCl}$.

We parametrise the corrugation term in the potential as

$$
V_{g}(x, y)=c_{0}+c_{2}\left(x^{2}+y^{2}\right)
$$

The matrix elements of the corrugation term can be cast in the analytic form as

$$
\begin{aligned}
V_{m, n, G}^{m^{\prime}, n^{\prime}, G^{\prime}} & =<m, n ; k+G\left|2 V_{g}(x, y) \cos (g z)\right| m^{\prime}, n^{\prime} ; k+G^{\prime}> \\
& =\delta_{G, G^{\prime} \pm g}\left\{\left[c_{0}+\frac{c_{2}}{\delta^{2}}\left(m^{\prime}+n^{\prime}+1\right)\right] \delta_{m, m^{\prime}} \delta_{n, n^{\prime}}\right. \\
& +\frac{c_{2}}{2 \delta^{2}}\left[\delta_{n, n^{\prime}}\left(\sqrt{\left(m^{\prime}+1\right)\left(m^{\prime}+2\right)} \delta_{m, m^{\prime}+2}+\sqrt{m^{\prime}\left(m^{\prime}-1\right)} \delta_{m, m^{\prime}-2}\right)\right. \\
& \left.\left.+\delta_{m, m^{\prime}}\left(\sqrt{\left(n^{\prime}+1\right)\left(n^{\prime}+2\right)} \delta_{n, n^{\prime}+2}+\sqrt{n^{\prime}\left(n^{\prime}-1\right)} \delta_{n, n^{\prime}-2}\right)\right]\right\} .
\end{aligned}
$$

The parameters of the proposed model of interaction potential can be fixed by examining the potential obtained as a pairwise sum of binary He-C site interactions. We model the effective He-C site interaction with a Lennard-Jones (LJ) form, $4 \epsilon\left[(\sigma / r)^{12}-(\sigma / r)^{6}\right]$, where $r=|\mathbf{r}|$ denotes the distance between $\mathrm{He}$ atom and a $\mathrm{C}$ site. Various different forms of effective He-C potential, based on the analysis of He-graphite interaction, have been proposed in the literature 17, 18. The most important deficiency of the LJ form is the assumption that 
the potential depends only on the magnitude of r.7. However, in the view of a lack of knowledge on the precise geometry of a bundle, the LJ form should serve well in obtaining the main features of the interaction potential. The LJ parameters we adopt are $\epsilon=1.34$ meV, $\sigma=2.75 \AA$. This choice of parameters was suggested in Ref. 19, where the LJ form of the potential has been optimized to reproduce the experimental data on ${ }^{4} \mathrm{He}$ scattering from the basal (1000) plane of graphite. These parameters are slightly different from the ones reported in Ref. $5(\epsilon=1.46 \mathrm{meV}, \sigma=2.98 \AA)$ which were obtained from the semiempirical combining rules $\$$.

In the following, we consider $(10,10)$, "armchair" carbon nanotubes 11 with a diameter of $d=13.8 \AA$. The centers of the nanotubes are separated by $13.8 \AA+3.2 \AA=17.0 \AA$, where the value of the intertube separation of $3.2 \AA$ has been adopted, in agreement with experimental findings 14 and also with Refs. 1 and 5. Assuming that all the tubes surrounding a channel are axially aligned, and that the whole structure of the bundle can be obtained from a single tube by applying a two dimensional translation characterised by a general two dimensional lattice vector given as $\mathbf{t}=i \mathbf{t}_{1}+j \mathbf{t}_{2}$, where $i$ and $j$ are integers and $\mathbf{t}_{1}$ and $\mathbf{t}_{2}$ are basis vectors of a triangular lattice of the bundle, the parameters consistent with our model of the potential are $D=-36.8 \mathrm{meV}, \alpha=44.7 \mathrm{meV} / \AA^{2}, c_{0}=-0.05 \mathrm{meV}, c_{2}=10.7 \mathrm{meV} / \AA^{2}$ and $g=2 \pi / 2.5 \AA=2.511 / \AA$. This set of parameters provides an excellent fit to the angular average of the total potentiall.

The density of states per unit length of the channel can be obtained from

$$
g(E)=\frac{1}{L_{z}} \sum_{\lambda, k} \delta\left(E-E_{\lambda}(k)\right) .
$$

Some care should be taken in evaluation of Eq. (9) to properly account for the degeneracy present in the energy spectrum, $E_{\lambda}(k)$. In Fig. 1 we present the band structure and the corresponding density of states obtained from the determined set of potential parameters. For convenience of presentation, the delta functions in equation (9) have been broadened to gaussians of width $0.03 \mathrm{meV}$. Note the enhancement of the lowest band effective mass of He atom, $M^{*}=2.37 M=9.5 \mathrm{amu}$ and the band gap between $-27.75 \mathrm{meV}$ and $-25.5 \mathrm{meV}$. 
The bandwidth of the lowest energy band is $0.19 \mathrm{meV}(2.2 \mathrm{~K})$. This should be compared with the value of $0.18 \mathrm{~K}$ found for $(18,0)$ tubes in Ref. 3. The difference between these two values is determined in part by the different periodicities (cell lengths) of the "zigzag" and "armchair" carbon nanotubes.

The mass enhancement should be compared with the one found by authors of Ref. 2, $M^{*}=1.3 M$. The difference between their and our value can be explained by somewhat larger intertube separation presumed in the calculations in Ref. 2 (3.3 $\AA$, whereas we used $3.2 \AA)$. As shown in Ref. 2 this parameter strongly influences the magnitude of the potential corrugation and thus also the effective mass enhancement. The ground-state energy, $E_{\Gamma}$, of adsorbed ${ }^{4} \mathrm{He}$ atoms as found from our model potential is $-27.94 \mathrm{meV}$. The ground-state energy found in references 4 and 5 for nanotubes of the same diameter $(13.8 \AA)$, but for the interaction potential which does not exhibit corrugation along the channel axis was -29.07 $\mathrm{meV}$ and $-33.3 \mathrm{meV}$, respectively. This shows consistence of our potential with the potentials previously proposed in the literature. The experimental estimate of the binding energy based on desorption measurements was reported to be $-28.5 \mathrm{meV}$, in good agreement with the value we obtain. The subsequent reevaluationed of the experimental data first presented in Ref. 7 yielded a smaller binding energy $(-19.8 \mathrm{meV})$. The authors of Ref. 21 suggested that this value of binding energy may be compatible with adsorption of He on the outer grooves of the bundle. Our results should be of help to determine the relevant adsorption sites.

One of the attractive features of our potential model is that the various different arrangements of nanotubes surrounding a channel can be easily (but approximately) simulated by changing the parameters of the potential, especially those representing the corrugation of the potential. Additionally, the effects of possible inadequacies of the binary He-C site potential (such as the lack of anisotropy in the effective interaction) can be avoided by treating the parameters $c_{0}$ and $c_{2}$ of the potential as variables. In Fig. 2 we present the behavior of the density of states as a function of parameter $c_{2}$ which defines the magnitude of the potential corrugation. As expected, for smoother potentials, the band gaps, binding energy and effective mass are smaller. In particular, the lowest band mass enhancements are 2.37, 
1.23 and 1.02 for the calculations presented in panels a), b) and c) of Fig. 2, respectively.

\section{SPECIFIC HEAT OF LOW DENSITY ${ }^{4}$ He INTERSTITIAL GAS}

Knowing the density of states, we can calculate the isosteric specific heat of He atoms adsorbed in the nanotube interstitial channels. The approach we pursue is similar to the calculation of specific heat of the adsorbed (sub)monolayer of noninteracting atoms on a corrugated surface discussed in Ref. 15. The influence of adsorbate-adsorbate interactions on the specific heat of the He overlayer on graphite has been studied in Ref. 22.

The total energy of a system of $\mathrm{N}$ noninteracting ${ }^{4} \mathrm{He}$ atoms is given by

$$
U=L_{z} \int_{E_{\Gamma}}^{\infty} g(E) E f(E, T) d E
$$

The Bose-Einstein distribution function in the above equation is given by

$$
f(E, T)=\frac{1}{\exp \left(\frac{E-\mu}{k_{B} T}\right)-1},
$$

where $k_{B}$ is the Boltzmann constant and $T$ is temperature. The chemical potential $\mu$ can be determined from the requirement of the conservation of number of adsorbates, $N$,

$$
N=L_{z} \int_{E_{\Gamma}}^{\infty} g(E) f(E, T) d E .
$$

Solution of Eq. (12) results in the temperature dependence of chemical potential $\mu$ for a fixed total number of ${ }^{4} \mathrm{He}$ atoms. The isosteric specific heat $C / N$ can be obtained from

$$
\frac{C}{N}=\frac{1}{N}\left(\frac{d U}{d T}\right)_{N}=L_{z} \frac{k_{B}}{\left(k_{B} T\right)^{2}} \frac{I_{2}-I_{1}^{2} / I_{0}}{N},
$$

where integral quantities $I_{j}$ are defined as

$$
I_{j}=\int_{E_{\Gamma}}^{\infty} g(E) E^{j} \exp \left[\frac{E-\mu(T)}{k_{B} T}\right] f^{2}(E, T) d E, j=0,1,2,
$$

in accordance with the notation of Ref. [15. Equations (13) and (14) are very convenient for numerical implementation once the dependence of chemical potential on temperature, $\mu(T)$ has been found from Eq. (12). 
In Fig. 3 we plot the isosteric specific heat as a function of temperature for three different average adsorbate linear densities and with potential parameters obtained from the assumption of perfect alignment of the tubes. The dashed (full) line corresponds to average interadsorbate distance of $1 \mathrm{~nm}(2 \mathrm{~nm})$. It is interesting to note here that the mean He-He distance along the channel estimated from experimental data in Ref. 7 was found to be about $3 \mathrm{~nm}$. The dash-dotted line in Fig. 3 corresponds to this average linear ${ }^{4} \mathrm{He}$ density.

As noted in Ref. 2, perfectly aligned tubes result in the highest corrugation of the potential seen by He adsorbate. In this respect, it is interesting to see how the specific heat changes as a function of a potential corrugation along the channel axis. In Fig. Đ we plot the variation of specific heat with temperature for three different corrugation parameters $c_{2}$ and we fix the average linear density of He adsorbates to $N / L_{z}=0.11 / \AA$. All three curves display a nonmonotonic behavior and a minimum in specific heat around $T=4 \mathrm{~K}$ is observed. At higher temperatures all curves approach to the value of 0.5 (thin dotted line), reflecting a specific heat characteristic of a particle with one translational degree of freedom. For even higher temperatures $(\sim 20 \mathrm{~K}$, not shown in the figure $)$ the excitation of higher harmonic oscillator levels $\left[m^{\prime}\right.$ or $n^{\prime}$ in Eq. (6) different from zero] becomes possible and the specific heat becomes larger than 0.5. For such high temperatures, desorption of He from the sample becomes probable 6 . The minimum in specific heat occurs due to the existence of the band gap in the density of states 15.8 .

As the band gap becomes smaller (i.e. for lower corrugations of the potential) the minimum in specific heat becomes less pronounced and practically disappears for the smallest corrugation of the potential considered in the calculations presented in Fig. \$. It is interesting to note that our results show similar trends as the ones presented in Ref. 15. Due to the different dimensionality in our case, the specific heat is a factor of about 2 smaller than the one calculated for rare overlayer of He atoms on graphite 15 . Also, due to the effective magnification of the corrugation in a restricted geometry of the interstitial channel, the variation of the specific heat with temperature is significantly more pronounced. 


\section{SUMMARY AND CONCLUSIONS}

A robust prediction of our model is appearance of band gaps in the density of states of He adsorbates in the interstitial channels. This prediction is based on the assumption of periodicity of the potential along the channel axis and it holds irrespective of the precise alignment of the tubes surrounding a channel, as long as all three tubes have the same internal symmetry. The existence of the band gap causes a nonmonotonic behavior of the specific heat associated with the interstitial He gas, an effect previously observed for low-density overlayer of He atoms on a graphite surface 5 . We observe an appearance of the minimum in specific heat around $T=4 \mathrm{~K}$. As the corrugation of the potential along the channel axis smoothens, the minimum in the specific heat becomes less dramatic and ultimately vanishes. The effects we predict should be easily tested experimentally in the regimes where the specific heat of the adsorbates can be easily distinguished from the overall specific heat (for low temperatures and not too low concentrations of He adsorbates 10 ).

\section{ACKNOWLEDGEMENTS}

A. Š. acknowledges stimulating discussions with K. Biljaković and J.C. Lasjaunias. 


\section{REFERENCES}

${ }^{1}$ M.M. Calbi, M.W. Cole, S.M. Gatica, M.J. Bojan, and G. Stan, Rev. Mod. Phys. 73, 857 (2001)

${ }^{2}$ M. Boninsegni, S.-Y. Lee, and V.H. Crespi, Phys. Rev. Lett. 86, 3360 (2001)

${ }^{3}$ M.W. Cole, V.H. Crespi, G. Stan, C. Ebner, J.M. Hartman, S. Moroni, and M. Boninsegni, Phys. Rev. Lett. 84, 3883 (2000)

${ }^{4}$ G. Stan, V.H. Crespi, M.W. Cole, and M. Boninsegni, J. Low Temp. Phys. 113, 447 (1998)

${ }^{5}$ G. Stan, M.J. Bojan, S. Curtarolo, S.M. Gatica, and M.W. Cole, Phys. Rev. B 62, 2173 (2000)

${ }^{6}$ G. Stan and M.W. Cole, Surf. Sci. 395, 280 (1997)

${ }^{7}$ W. Teizer, R.B. Hallock, E. Dujardin, and T.W. Ebbesen, Phys. Rev. Lett. 82, 5305 (1999)

${ }^{8}$ L.W. Bruch, M.W. Cole, and E. Zaremba, Physical Adsorption: Forces and Phenomena, Clarendon Press, Oxford, 1997

${ }^{9}$ J. Hone, B. Batlogg, Z. Benes, A.T. Johnson, and J.E. Fischer, Science 289, 1730 (2000)

${ }^{10}$ J.C. Lasjaunias, K. Biljaković, Z. Benes, J.E. Fischer, and P. Monceau, Phys. Rev. B 65, $113409(2002)$

${ }^{11}$ N. Hamada, S.-I. Sawada, and A. Oshiyama, Phys. Rev. Lett. 68, 1579 (1992)

12 J.W.G. Wildoer, L.C. Venema, A.G. Rinzler, R.E. Smalley, and C. Dekker, Nature 391, $59(1998)$

${ }^{13}$ E. Anglaret, S. Rols, and J.-L. Sauvajol, Phys. Rev. Lett. 81, 4780 (1998)

${ }^{14}$ A. Thess, R. Lee, P. Nikolaev, H.J. Dai, P. Petit, J. Robert, C.H. Xu, Y.H. Lee, S.G. Kim, 
A.G. Rinzler, D.T. Colbert, G.E. Scuseria, D. Tomanek, J.E. Fischer, and R.E. Smalley, Science 273, 483 (1996)

${ }^{15}$ W.E. Carlos and M.W. Cole, Phys. Rev. B 21, 3713 (1980)

${ }^{16}$ M.C. Vargas and W.L. Mochán, Surf. Sci. 355, 115 (1996)

${ }^{17}$ M.W. Cole, D.R. Frankl, and D.L. Goodstein, Rev. Mod. Phys. 53, 199 (1981)

${ }^{18}$ F. Toigo and M.W. Cole, Phys. Rev. B 32, 6989 (1985)

${ }^{19}$ N. Garcia, W.E. Carlos, M.W. Cole, and V. Celli, Phys. Rev. B 21, 1636 (1980)

${ }^{20}$ W. Teizer, R.B. Hallock, E. Dujardin, and T.W. Ebbesen, Phys. Rev. Lett. 84, 1844 $(2000)$

${ }^{21}$ Y.H. Kahng, R.B. Hallock, E. Dujardin, and T.W. Ebbesen, J. Low Temp. Phys. 126, $223(2002)$

${ }^{22}$ R.L. Siddon and M. Schick, Phys. Rev. A 9, 907 (1974) 


\section{FIGURES}

FIG. 1. a) Calculated band structure of a ${ }^{4} \mathrm{He}$ atom adsorbed in the interstitial channel. b) The corresponding density of states per unit length.

FIG. 2. Density of states of ${ }^{4} \mathrm{He}$ atoms adsorbed in the interstitial channel as a function of the magnitude of corrugation of interaction potential ( $c_{2}$ parameter). a) $c_{0}=-0.05 \mathrm{meV}, c_{2}=10.7$ $\mathrm{meV} / \AA^{2}$. b) $c_{0}=-0.05 \mathrm{meV}, c_{2}=5 \mathrm{meV} / \AA^{2}$. c) $c_{0}=-0.05 \mathrm{meV}, c_{2}=2 \mathrm{meV} / \AA^{2}$.

FIG. 3. Isosteric specific heat of ${ }^{4} \mathrm{He}$ gas within the nanotube interstitial channels as a function of the sample temperature for three different average He linear densities, $N / L_{z}$. Dash-dotted line: $N / L_{z}=0.0331 / \AA$. Full line: $N / L_{z}=0.051 / \AA$. Dashed line: $N / L_{z}=0.11 / \AA$.

FIG. 4. Dependence of specific heat of ${ }^{4} \mathrm{He}$ gas on the corrugation of the interaction potential along the channel axis. Full line: $c_{0}=-0.05 \mathrm{meV}, c_{2}=10.7 \mathrm{meV} / \AA^{2}$. Dashed line: $c_{0}=-0.05$ $\mathrm{meV}, c_{2}=5 \mathrm{meV} / \AA^{2}$. Dash-dotted line: $c_{0}=-0.05 \mathrm{meV}, c_{2}=2 \mathrm{meV} / \AA^{2}$. In these calculations, average linear density of ${ }^{4} \mathrm{He}$ atoms was set to $0.11 / \AA$. 


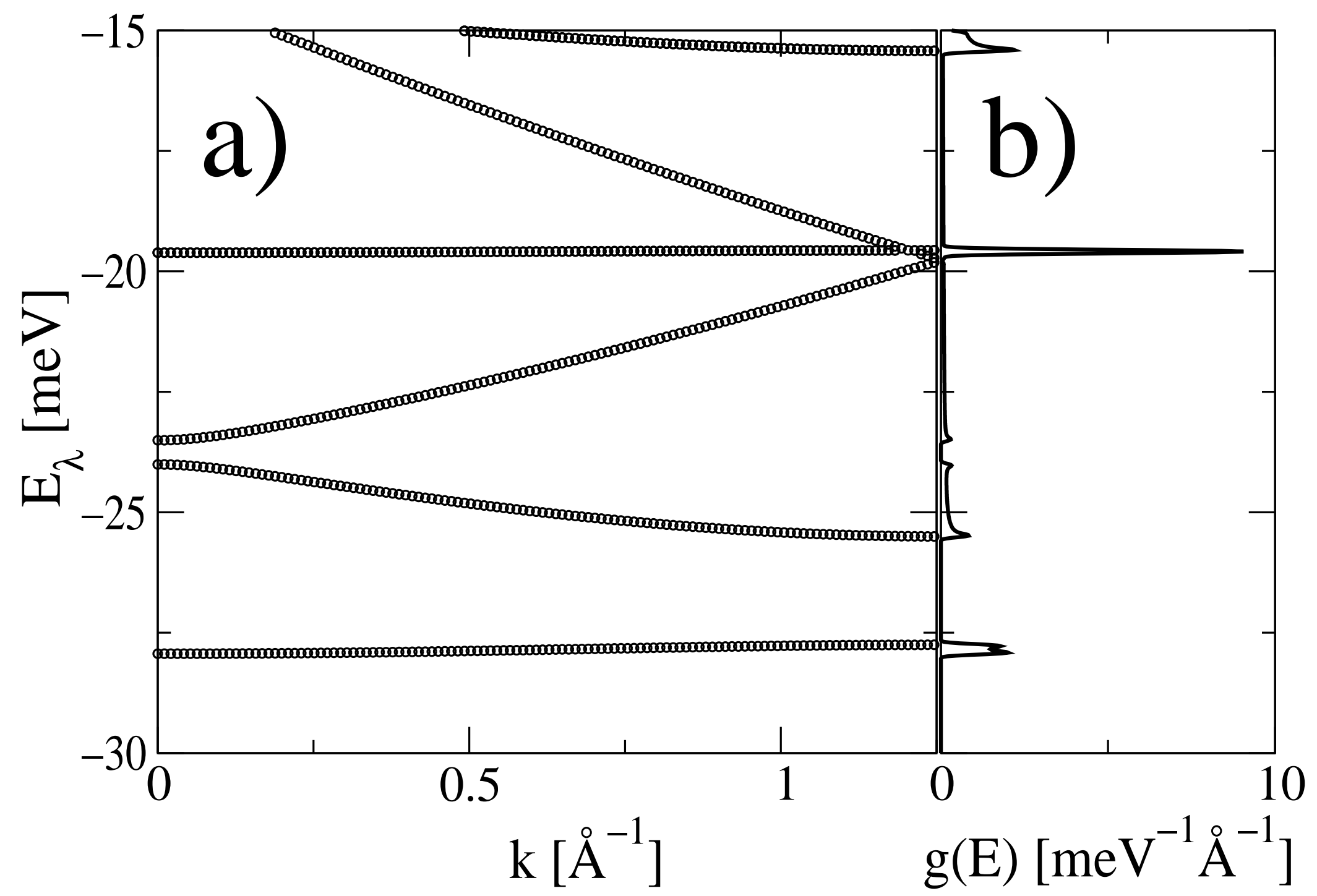




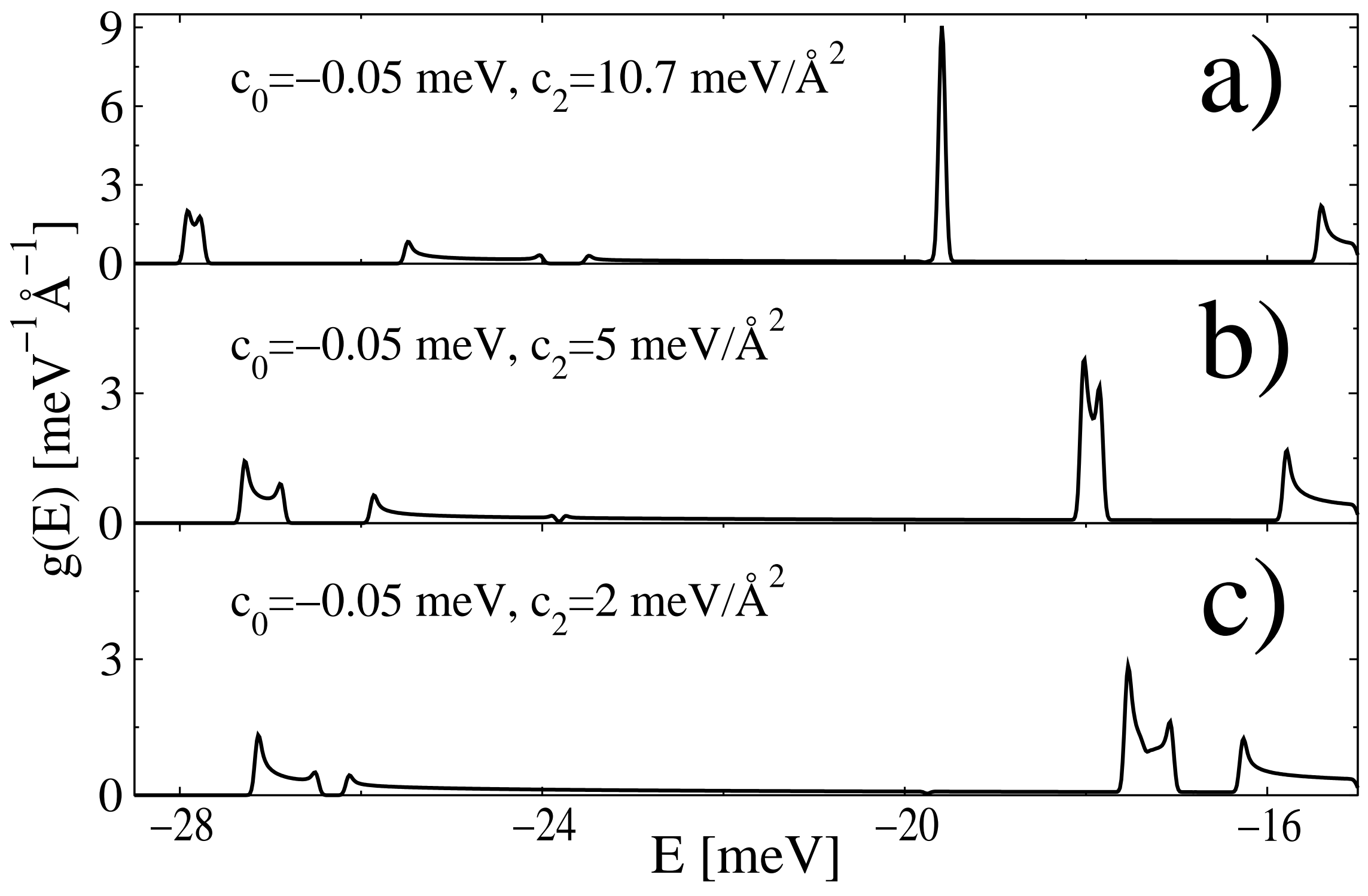




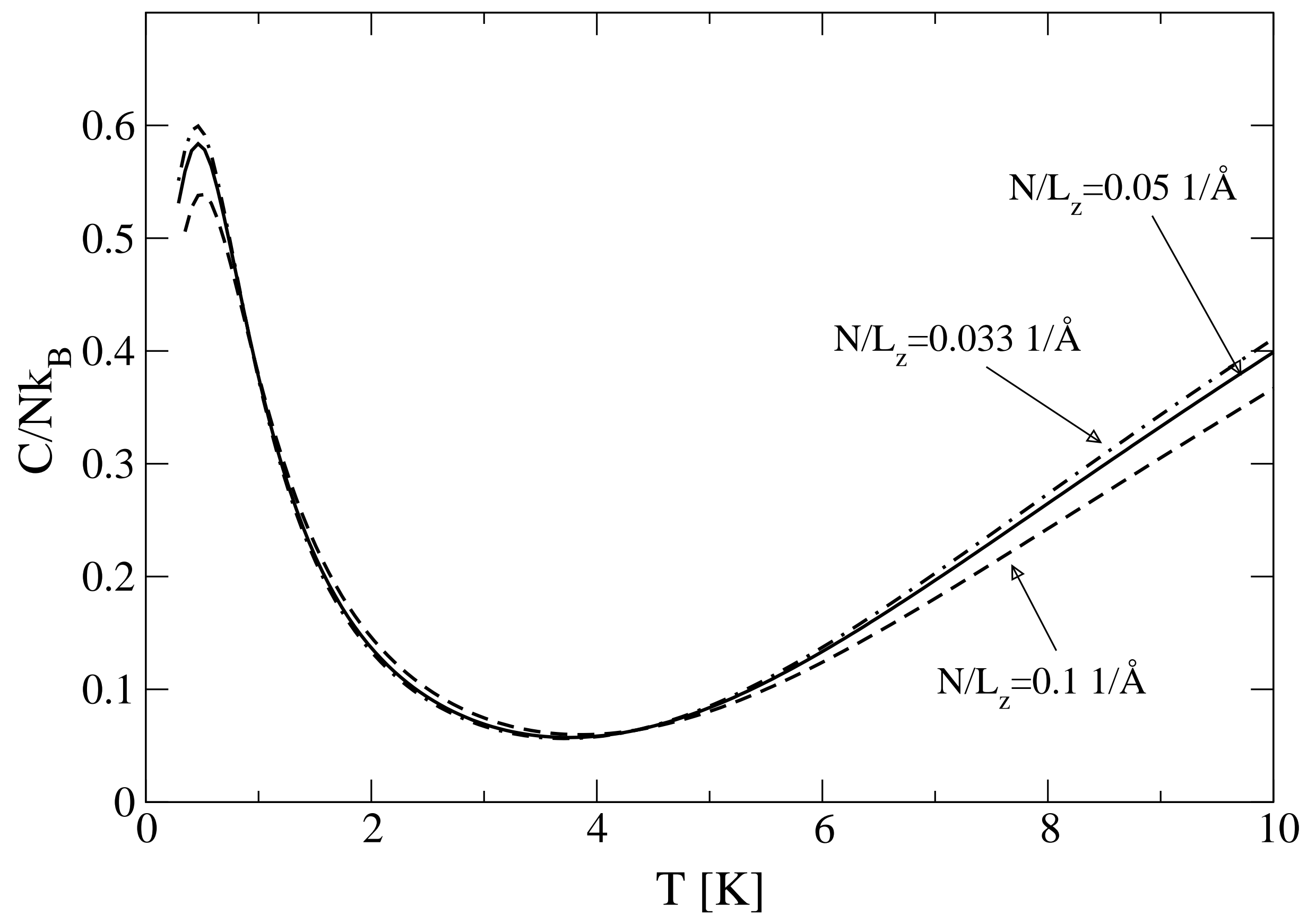




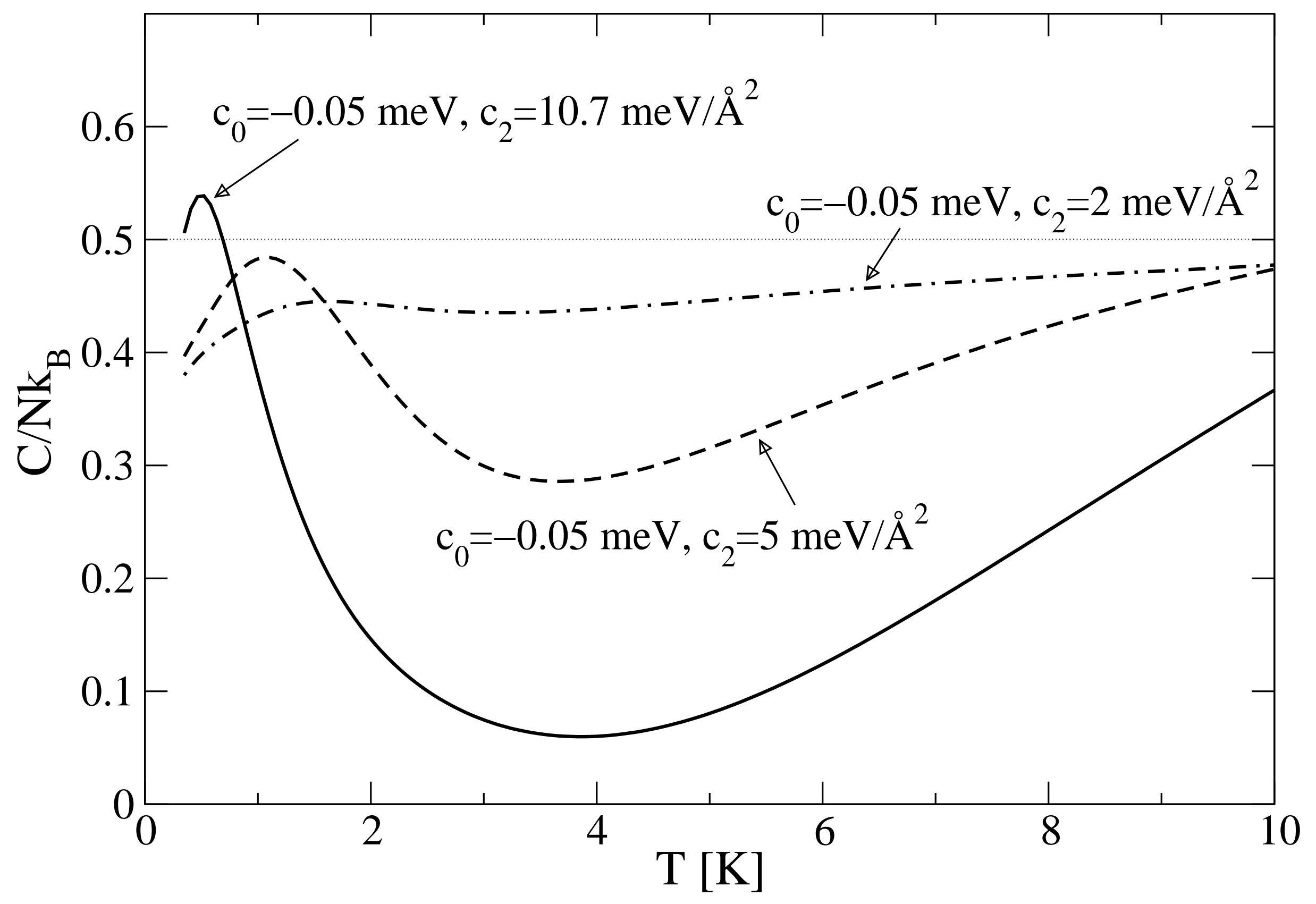

